\section{Epidermal Transplantation during Chemical Carcinogenesis}

IN the study of the mechanism of epidermal carcinogenesis, it is perhaps not surprising that attention has been mainly directed to the epithelium of the epidermis itself. Nevertheless, changes occur in the dermis and subcutis which can be distinguished from the effects of non-specific irritants, and it has been suggested that these changes are of importance in the carcinogenic reaction ${ }^{1}$. As it is now technically possible to make pure epidermal grafts ${ }^{2}$, the opportunity has been taken to test this hypothesis by transferring the epidermis of a carcinogen-treated site to an untreated body-site, and vice versa.

Mice were painted with methylcholanthrene $(0.3$ per cent in acetone) once a week for twelve weeks on the skin of the right scapular region (referred to as the 'treated area'). They were then left untreated for two weeks, the few animals with tumours discarded, and the remainder subjected to one of the following operations :

(a) Epidermis removed from treated area, to which tail skin epidermis was transplanted.

(b) Epidermal grafts from treated area transplanted to a bed prepared by removing the epidermis from the left dorsal thorax (referred to as the 'grafted area').

(c) Thiersch grafts (that is, epidermis and superficial dermis) from treated area transplanted to grafted area (single bed).

(d) Thiersch grafts of treated area transplanted to four separate beds in grafted area.

Results of experiments :

(a) Of 21 survivors when the first tumour appeared in this group, 12 have developed tumours on the treated area in an average time of $78 \pm 17$ days after operation.

(b) 28 survivors at first tumour : 13 tumours on the treated area in $93 \pm 16$ days; no tumours on the grafted area.

(c) 7 survivors; 4 tumours in $92 \pm 45$ days ( 3 of these definitely on the treated area, the other doubtful in position). In addition, one of the mice had a nodule on the grafted area, which had the histological structure of an implantation epidermoid cyst.

(d) 8 survivors; 5 tumours in $67+37$ days, all on the treated area. No tumours on the grafted area.

The validity of these results obviously depends on the grafts having 'taken' adequately. They appeared to have done so, but it is not yet possible to be as certain on this point in the mouse as it would be in larger rodents. The part played by the hair follicles has to be taken into consideration; the deep parts are necessarily left in situ during grafting, while the superficial parts are presumably transferred with the graft. Histological examination does not suggest that a considerable proportion of the tumours originated in hair follicles, and the negative results on the grafted area in experiment (b) may be significant.

This work was aided by funds from the Birmingham Branch of the British Empire Cancer Campaign.

R. E. BILLINGHam

J. W. ORR

D. L. Woodhouse

Departments of Pathology and Zoology, University of Birmingham. Oct. 11.

Orr, J. W., J. Path. Bact., 46, 495 (1938).

- Billingham, R. E., and Medawar, P. B. (unpublished); ef. Heredity,

\section{Estimation of Collagen in Hard Tissues}

IN analysing the protein constituents of connective tissues, it is usually assumed that collagen is repre. sented by a fraction of the total nitrogen which is precipitable by tannic acid after autoclaving the washed sample. The existing procedure for the determination of this protein in bone ${ }^{1}$ was applicd to dentine ${ }^{2}$, but later shown to be unsatisfactory. Preparation of the powdered sample by grinding results in the conversion of at least one-tenth of the collagen to gelatin. Drastic autoclaving conditions account for the failure to precipitate a similar fraction of the protein thus made soluble, while somewhat more of the gelatin remains adsorbed on the auto. claved dentine powder even after washing with boiling water. These facts became apparent when a purificd hide collagen sample ${ }^{3}$ was mixed with the correct proportion of the residue preparcd by extracting dentine with boiling alkaline ethylene glycol and analysed under the same conditions.

PERCENTAGes of TOTAL NITROGEN FOCND IN FRACTIONS OBTAINRD WHEN SAMPLES ARE WASHED AND THE GELATIN (RELEASED BY Autoclaving) Precipitated with TaNNic ACID

\begin{tabular}{|l|c|c|c|c|}
\hline \multicolumn{1}{|c|}{ Sample } & $\begin{array}{c}\text { Soluble } \\
\text { Soluter }\end{array}$ & $\begin{array}{c}\text { Soluble on } \\
\text { autoclaving } \\
\text { in water } \\
\text { Precipitate }\end{array}$ & $\begin{array}{c}\text { Filtrate } \\
\text { Adsorbed } \\
\text { on residue }\end{array}$ \\
\hline Dentine collagen & 0 & 91 & 8 & 0 \\
Hide collagen & 0 & 91 & 9 & 0 \\
Dentine collagen* & 0 & 81 & 7 & 12 \\
Hide collagen* & 0 & 82 & 7 & 12 \\
Crushed dentine* & 5 & 65 & 7 & 24 \\
Crushed dentine & 5 & 75 & 8 & 12 \\
Ground dentine & 15 & 66 & 6 & 13 \\
\hline
\end{tabular}

* Added to an equivalent quantity of deproteinized dentine.

The fractions thus obtained were then examined after hydrolysis by comparing their amino-acid 'spectra'. These were obtained by using a method" which has been applied here to the characterization of proteins. Ninhydrin-developed paper chromato. gram strips were passed through a photo-electrio colorimeter and the optical densities then plotted against the distances travelled by the amino-acids. Water-soluble protein from crushed dentine was thus shown to be gelatin. Included with the gelatin fraction adsorbed on the residue left after autoclaving was an insoluble protein, amounting to 1 per cent of the total collagen and giving a 'spectrum' which was not typical of collagen, elastin or reticulin; the residue from hide collagen has similarly been differentiated ${ }^{3}$.

Further evidence as to the amount of collagen in hard tissues is provided by a method which may have useful applications as it avoids both drying and weighing the samples to be analysed. Convenient volumes of dilute phosphoric acid solutions contain. ing dentine or the isolated collagen were digested with $(a)$ sulphuric acid, using a peroxide catalyst, and $(b)$ a dichromate-sulphuric acid reagel.t. From (a) the total nitrogen was estimated colorimetrically, and from $(b)$ the amount of oxidant consumed was calculated after iodometric titration. The ratio of $a$ to $b$ was expressed as ' $m g m$. nitrogen $/ \mathrm{ml}$. $N$ thiosulphate', and measured the relation between protein and total organic matter. Ratios obtained by analyses on deciduous dentine, on incisor, canine, premolar and molar permanent dentine, varied by less than I per cent from their mean, which was definitely less than the single ratio equally applicable to the isolated collagens from dentine, bone and ox hide. 